\title{
GROUP $C^{*}$-ALGEBRAS AS ALGEBRAS OF “CONTINUOUS FUNCTIONS" WITH NON-COMMUTING VARIABLES
}

\author{
WOJCIECH SZYMAŃSKI
}

(Communicated by John B. Conway)

\begin{abstract}
It is shown that a system of commutation relations depending on the structure constants of a Lie algebra $\mathfrak{g}$ leads to a $C^{*}$-algebra which is isomorphic to the group $C^{*}$-algebra of the simply connected Lie group associated with $\mathfrak{g}$.
\end{abstract}

\section{INTRODUCTION}

Let $\mathbf{G}$ be a connected simply connected Lie group, $\mathfrak{g}$ be the corresponding Lie algebra (of the right invariant vector fields), $X_{1}, \ldots, X_{N}$ form a basis in $\mathfrak{g}$ such that

$$
\left[X_{m}, X_{n}\right]=\sum_{k=1}^{N} c_{m n}^{k} X_{k}
$$

If $U$ is a strongly continuous unitary representation of $\mathbf{G}$ acting on $\mathscr{H}$ and $d U$ the infinitesimal representation associated with $U, A_{j}=\overline{d U\left(X_{j}\right)}$, then we define operators $B_{j}$ for $j=0,1, \ldots, N$ as

$$
\begin{aligned}
& B_{0}=\left(1+\sum_{k=1}^{N} A_{k}^{*} A_{k}\right)^{-1}, \\
& B_{j}=A_{j} B_{0} .
\end{aligned}
$$

Received by the editors February 8, 1988.

1980 Mathematics Subject Classification (1985 Revision). Primary 46L99; Secondary 22D25.

Key words and phrases. Compact domain, group $C^{*}$-algebra, Lie algebra of a Lie group, continuous operator function. 
It is easy to establish that $B_{j}$ are bounded operators and satisfy the following conditions:

$$
\begin{gathered}
B_{0}^{*}=B_{0}, \\
B_{m}^{*}=-B_{0} B_{m}, \quad m=1, \ldots, N, \\
B_{0}\left(1-B_{0}\right)=\sum_{k=1}^{N} B_{k}^{*} B_{k}, \\
B_{n}^{*} B_{m}-B_{m}^{*} B_{n}=\sum_{k=1}^{N} c_{m n}^{k} B_{0} B_{k}, \quad m, n=1, \ldots, N, \\
B_{m}^{*}+B_{m}=\sum_{k, r=1}^{N} c_{m k}^{r}\left(B_{r}^{*} B_{k}+B_{k}^{*} B_{r}\right), \quad m=1, \ldots, N .
\end{gathered}
$$

The second formula is a consequence of antisymmetry of $A_{m}$, the fourth we get considering the commutator $\left[X_{m}, X_{n}\right]$ and the fifth similarly computing $\left[X_{m}, \Delta\right]\left(\Delta=\sum_{k=1}^{N} X_{k}^{2}\right.$ in the enveloping algebra).

Starting from the above relations we shall construct a $C^{*}$-algebra which will appear to be isomorphic with $C^{*}(\mathbf{G})$.

I take this opportunity to thank Prof. S. L. Woronowicz who suggested the problem and gave much helpful advice.

\section{CONSTRUCTION OF THE ALGEBRA}

The construction is an application of the method shown in [1]. We shall also keep the notation of that paper.

There was introduced a notion of a compact domain there as a system of $N$-tuples of bounded operators. More precisely the family $D$ of sets $D(\mathscr{H}) \subseteq$ $\mathscr{B}(\mathscr{H})^{N}$ (where $\mathscr{H}$ denotes any separable Hilbert space) is called a compact domain if for every separable Hilbert space the following three conditions are fulfilled:

(1) For every $a \in D(\mathscr{H})$ and every $*$-representation

$$
\pi: C^{*}(a) \rightarrow \mathscr{B}(\mathscr{K})
$$

we have $\pi(a) \in D(\mathscr{K})$;

(2) If for a given $a \in \mathscr{B}(\mathscr{H})^{N}$ there exists a family $\left\{\pi_{t}\right\}_{t \in T}$ of representations of $C^{*}(a)$ such that $\bigcap_{t \in T} \operatorname{Ker} \pi_{t}=\{0\}$ and $\pi_{t}(a) \in D\left(\mathscr{H}_{t}\right)$ for all $t \in T$ (where $\mathscr{H}_{t}$ denotes the carrier Hilbert space of $\pi_{t}$ ) then $a \in D(\mathscr{H})$;

(3) There exists a constant $M>0$ independent of $\mathscr{H}$ such that

$$
\sup _{a \in D(\mathscr{P})}\left\|a_{k}\right\| \leq M \text {. }
$$


If $a \in \mathscr{B}(\mathscr{H})^{N}$ and $a=\left(a_{1}, \ldots, a_{N}\right)$ then $C^{*}(a)$ denotes the unital $C^{*}$ algebra generated by $a_{1}, \ldots, a_{N}$. Similarly if $\pi$ is a representation of $C^{*}(a)$ then $\pi(a)$ denotes $\left(\pi\left(a_{1}\right), \ldots, \pi\left(a_{N}\right)\right)$.

Let $C(D)$ be the unital $C^{*}$-algebra of continuous functions on $D . F$ is an element of $C(D)$ if for any Hilbert space $\mathscr{H}$ and any $\left(a_{1}, \ldots, a_{N}\right) \in D(\mathscr{H})$, $F\left(a_{1}, \ldots, a_{N}\right)$ lies in $C^{*}(a)$, and for any representation $\pi$ of $C^{*}(a)$ we have

$$
F\left(\pi\left(B_{0}\right), \ldots, \pi\left(B_{N}\right)\right)=\pi\left(F\left(B_{0}\right), \ldots, F\left(B_{N}\right)\right) .
$$

For any separable Hilbert space $\mathscr{H}$ we set $\mathbf{D}(\mathscr{H})=\left\{\left(B_{0}, \ldots, B_{N}\right) \in\right.$ $\mathscr{B}(\mathscr{H})^{N+1}: B_{0}, \ldots, B_{N}$ satisfy conditions (1)-(5)\}.

By virtue of [1, Theorem 1.3] we get

Proposition. $\mathbf{D}=\{\mathbf{D}(\mathscr{H}): \mathscr{H}$-separable Hilbert space $\}$ is a compact domain.

We define $C_{0}(\mathbf{D})$ as those elements $F$ belonging to $C(\mathbf{D})$ for which $F(0, \ldots, 0)=0 . C_{0}(\mathbf{D})$ is clearly a $C^{*}$-subalgebra of $C(\mathbf{D})$ (without unity). We shall prove the following

Theorem. There is a natural ${ }^{*}$-isomorphism of the group $C^{*}$-algebra $C^{*}(\mathbf{G})$ onto $C_{0}(\mathbf{D})$.

Continuous functions on a compact space vanishing at a single point form a commutative $C^{*}$-algebra which topologically corresponds to a locally compact space. One can now view at the group $C^{*}$-algebra of a simply connected lie group as a noncommutative $C^{*}$ algebra of continuous functions on a locally compact domain vanishing at a single "point".

We precede the proof of the theorem with two lemmas.

Lemma 1. Let $\mathscr{H}$ be a Hilbert space and $\left(B_{0}, \ldots, B_{N}\right)$ be operators in $\mathscr{B}(\mathscr{H})$ satisfying conditions (1)-(5). Assume that $\operatorname{ker} B_{0}=\{0\}$. Then there exists a strongly continuous unitary representation $U$ of the group $\mathbf{G}$ acting on $\mathscr{H}$ such that

$$
\overline{d U\left(X_{m}\right)}=\overline{B_{m} B_{0}^{-1}}, \quad m=1, \ldots, N .
$$

Proof. Setting $A_{m}=\overline{B_{m} B_{0}^{-1}}$ for $m=1, \ldots, N$ and $\mathscr{D}=\operatorname{Dom}\left(B_{0}^{-1}\right)$ we shall check that the following conditions hold:

1. $A_{m}$ are skew-symmetric (i.e. $A_{m} \subseteq-A_{m}^{*}$ ) $, m=1, \ldots, N$;

2. $\mathscr{D} \subseteq \operatorname{Dom}\left(A_{m} A_{n}\right), m, n=1, \ldots, N$;

3. for any $\xi \in \mathscr{D}$ we have $A_{m} A_{n} \xi-A_{n} A_{m} \xi=\sum_{k=1}^{N} c_{m n}^{k} A_{k} \xi, m, n=$ $1, \ldots, N$

4. $\sum_{k=1}^{N} A_{k}^{2}$ is essentially selfadjoint on $\mathscr{D}$. 
1. If $\xi, \varphi \in \operatorname{Dom}\left(B_{0}^{-1}\right)$ then there exist $\tilde{\xi}$, $\tilde{\varphi}$ such that $B_{0} \tilde{\xi}=\xi, B_{0} \tilde{\varphi}=$ $\varphi$, hence;

$$
\begin{aligned}
\left(A_{m} \xi, \varphi\right) & =\left(B_{m} B_{0}^{-1} \xi, \varphi\right)=\left(B_{m} B_{0}^{-1} B_{0} \tilde{\xi}, B_{0} \tilde{\varphi}\right)=\left(B_{0} \tilde{\xi}, B_{0} \tilde{\varphi}\right) \\
& =\left(\tilde{\xi}, B_{m}^{*} B_{0} \tilde{\varphi}\right) \quad\left(\text { from }(2) \text {-the second condition on } B_{m}\right) \\
& =-\left(\tilde{\xi}, B_{0} B_{m} \tilde{\varphi}\right) \\
& =-\left(B_{0} \tilde{\xi}, B_{m} B_{0}^{-1} B_{0} \tilde{\varphi}\right)=-\left(\xi, B_{m} B_{0}^{-1} \varphi\right)=-\left(\xi, A_{m} \varphi\right) .
\end{aligned}
$$

2. We must show that $B_{n}(\mathscr{C}) \subseteq \operatorname{Dom}\left(\overline{B_{m} B_{0}^{-1}}\right)$. For any $k=1, \ldots, N$, $B_{k} B_{0}^{-1 / 2}$ is bounded. Indeed

$$
\begin{aligned}
\left\|\left(B_{k} B_{0}^{-1 / 2}\right) B_{0} \xi\right\|^{2} & =\left(B_{k}^{*} B_{k} B_{0}^{1 / 2} \xi, B_{0}^{1 / 2} \xi\right) \quad(\text { from (3)) } \\
& \leq\left(B_{0} B_{0}^{1 / 2} \xi, B_{0}^{1 / 2} \xi\right)=\left\|B_{0} \xi\right\|^{2} .
\end{aligned}
$$

Therefore $B_{0}^{-1 / 2} B_{k}^{*}$ is also bounded, so $B_{k}^{*}(\mathscr{H}) \subseteq \operatorname{Dom}\left(B_{0}^{-1 / 2}\right)$ and (5) now implies that $B_{n}(\mathscr{H}) \subseteq \operatorname{Dom}\left(B_{0}^{-1 / 2}\right)$. Hence $\overline{B_{m} B_{0}^{-1} B_{n}=}$ $\left(\overline{B_{m} B_{0}^{-1 / 2}}\right)\left(B_{0}^{-1 / 2} B_{n}\right)$ is everywhere defined (as a product of two bounded operators).

3. It is enough to show that for any $\xi, \varphi \in \mathscr{H}$

$$
\left(\left(A_{m} A_{n}-A_{n} A_{m}\right) B_{0} \xi, B_{0} \varphi\right)=\left(\sum_{k=1}^{N} c_{m n}^{k} A_{k} B_{0} \xi, B_{0} \varphi\right) .
$$

We know that $A_{m}, A_{n}$ are skew-symmetric and $B_{0} \varphi$ is in their domains, so

$$
\begin{aligned}
& \left(\left(A_{m} A_{n}-A_{n} A_{m}\right) B_{0} \xi, B_{0} \varphi\right)=-\left(A_{n} B_{0} \xi, A_{m} B_{0} \varphi\right)+\left(A_{m} B_{0} \xi, A_{n} B_{0} \varphi\right) \\
& \quad=-\left(\overline{B_{n} B_{0}^{-1}} B_{0} \xi, \overline{B_{m} B_{0}^{-1}} B_{0} \varphi\right)+\left(\overline{B_{m} B_{0}^{-1}} B_{0} \xi, \overline{B_{n} B_{0}^{-1}} B_{0} \varphi\right) \\
& \quad=\left(\left(-B_{m}^{*} B_{n}+B_{n}^{*} B_{m}\right) \xi, \varphi\right) .
\end{aligned}
$$

On the other hand

$$
\left(\sum_{k=1}^{N} c_{m n}^{k} A_{k} B_{0} \xi, B_{0} \varphi\right)=\left(\sum_{k=1}^{N} c_{m n}^{k} \overline{B_{k} B_{0}^{-1}} B_{0} \xi, B_{0} \varphi\right)=\left(\sum_{k=1}^{N} c_{m n}^{k} B_{0} B_{n} \xi, \varphi\right)
$$

and the equality holds (from (4)).

4. $\sum_{k=1}^{N} A_{k}^{2}$ is essentially selfadjoint on $\mathscr{D}$ because it equals

$$
I-\left.B_{0}^{-1}\right|_{\mathscr{D}}
$$

Now the proof follows from Nelson's integrability theorem [2. Corollary 9.1].

We are now able to prove the following basic lemma. 
Lemma 2. There exist elements $b_{0}, \ldots, b_{N}$ of $C^{*}(\mathbf{G})$ such that:

1. For any operators $B_{0}, \ldots, B_{N}$ (on a Hilbert space) satisfying conditions (1)-(5) there is a *-homomorphism $U$ of $C^{*}(\mathbf{G})$ onto the $C^{*}$-algebra generated by $B_{0}, \ldots, B_{N}$, such that

$$
U\left(b_{k}\right)=B_{k}, \quad k=0,1, \ldots, N .
$$

2. $b_{0}, \ldots, b_{N}$ generate $C^{*}(\mathbf{G})$.

Proof. Considering the left regular representation of $\mathbf{G}$ acting on $L^{2}(\mathbf{G})$ we know (cf. [3, Theorem 3.1]) that for any $X \in \mathfrak{g}$ the operator $X+I-\sum_{k=1}^{N} X_{k}^{2}$ (acting on smooth functions with compact support) is invertible and the inverse operator has a kernel in $L^{1}(\mathbf{G})$. We define $b_{0}$ and $p_{m}(m=1, \ldots, N)$ as the images of the kernels of $\left(\overline{I-\sum_{k=1}^{N} X_{k}^{2}}\right)^{-1}$ and $\left(\overline{X_{m}+I-\sum_{k=1}^{N} X_{k}^{2}}\right)^{-1}$ respectively under the canonical inclusion of $L^{1}(\mathbf{G})$ into $C^{*}(\mathbf{G})$. For each natural number $n$ let $\psi_{n}$ be the following function:

$$
\psi_{n}(x)=\left\{\begin{array}{ll}
\frac{1}{x^{2}} & \text { if } x \geq \frac{1}{n}, \\
n^{2} & \text { if } x<\frac{1}{n},
\end{array} \quad x \in \mathbf{R} .\right.
$$

Of course $\psi_{n}\left(\left|p_{m}\right|\right)$ is a multiplier on $C^{*}(\mathbf{G})$. We shall show that $\psi_{n}\left(\left|p_{m}\right|\right) p_{m}^{*} \times$ $\left(b_{0}-p_{m}\right)$ is a Cauchy sequence in $C^{*}(\mathbf{G})$ (its limit will be denoted by $\left.b_{m}\right)$. To do that it will be enough to prove that for any nondegenerate representation $U$ of $C^{*}(\mathbf{G})$ the sequence

$$
\left(U\left(\psi_{n}\left(\left|p_{m}\right|\right) p_{m}^{*}\left(b_{0}-p_{m}\right)\right)\right)^{*}=\left(U\left(b_{0}\right)-U\left(p_{m}\right)^{*}\right) U\left(p_{m}\right) \psi_{n}\left(\left|U\left(p_{m}\right)\right|\right)
$$

is norm convergent.

Because we can extend $U$ to a representation of the group $\mathbf{G}$, we may also consider operators $A_{1}, \ldots, A_{N}, B_{0}, \ldots, B_{N}$ constructed for $U$ as in the introduction. It is easy to establish that $U\left(b_{0}\right)=B_{0}$ and $U\left(p_{m}\right)=\left(\overline{A_{m}+B_{0}^{-1}}\right)^{-1}$. We denote $U\left(p_{m}\right)$ by $P_{m}$.

By virtue of [2, Theorem 6.3] there exists a positive constant $M$ such that $\left\|P_{m}^{*-1} \xi\right\| \leq M^{2}\left\|B_{0}^{-1} \xi\right\|$ for any $\xi \in \mathscr{E}_{\infty}$ (where $\mathscr{E}_{\infty}$ are the smooth vectors of $U)$. So $\left\|B_{0} \xi\right\| \leq M^{2}\left\|P_{m} \xi\right\|=M^{2}\left\|P_{m} \mid \xi\right\|$ and operator monotony of the square root function implies that $\left\|B_{0}^{1 / 2} \xi\right\| \leq M\left\|\left|P_{m}\right|^{1 / 2} \xi\right\|$ for any $\xi \in \mathscr{H}$ ( $\mathscr{H}$-the carrier Hilbert space of $U$ ). In the proof of the previous lemma we showed that $B_{m}^{*} B_{0}^{-1 / 2}$ is bounded, so there is $\widetilde{C}>0$ such that for all $\xi$ in $\mathscr{H}$ we have $\left\|B_{m}^{*} \xi\right\| \leq \widetilde{C}\left\|B_{0}^{1 / 2} \xi\right\|$. Therefore $\left\|B_{m}^{*} \xi\right\| \leq \widetilde{C}\left\|B_{0}^{1 / 2} \xi\right\| \leq M \widetilde{C}\left\|\left|P_{m}\right|^{1 / 2} \xi\right\|$. We write $C$ instead of $M \widetilde{C}$.

If we take $\xi$ from $P_{m}^{*-1}\left(\mathscr{E}_{\infty}\right)$ then

$$
\left(P_{m}^{*}-B_{0}\right) \xi=B_{0}\left(B_{0}^{-1}-P_{m}^{*-1}\right) P_{m}^{*} \xi=B_{0} A_{m} P_{m}^{*} \xi=-B_{m}^{*} P_{m}^{*} \xi
$$


hence

$$
\begin{aligned}
B_{m}^{*} P_{m}^{*} & =B_{0}-P_{m}^{*}, \\
\left(U\left(b_{0}\right)-U\left(p_{m}\right)^{*}\right) U\left(p_{m}\right) \psi_{n}\left(\left|U\left(p_{m}\right)\right|\right) & =\left(B_{0}-P_{m}^{*}\right) P_{m} \psi_{n}\left(\left|P_{m}\right|\right) \\
& =B_{m}^{*}\left|P_{m}\right|^{2} \psi_{n}\left(\left|P_{m}\right|\right)=B_{m}^{*} \varphi_{n}\left(\left|P_{m}\right|\right),
\end{aligned}
$$

where $\varphi_{n}$ is a real function such that $\varphi_{n}(x)=1$ if $x>\frac{1}{n}$ and $\varphi_{n}(x)=n^{2} x^{2}$ if $x \leq \frac{1}{n}$. For any $\xi \in \mathscr{H}$ we have:

$$
\begin{aligned}
\left\|B_{m}^{*} \varphi_{n}\left(\left|P_{m}\right|\right) \xi-B_{m}^{*} \xi\right\| & =\left\|B_{m}^{*}\left(\varphi_{n}-1\right)\left(\left|P_{m}\right|\right) \xi\right\| \\
& \leq C\left\|\left|P_{m}\right|^{1 / 2}\left(\varphi_{n}-1\right)\left(\left|P_{m}\right|\right) \xi\right\| \leq C n^{-1 / 2}\|\xi\| .
\end{aligned}
$$

Therefore $B_{m}^{*} \varphi_{n}\left(\left|P_{m}\right|\right) \stackrel{\|\|}{\rightarrow} B_{m}^{*}$, so the elements $b_{1}, \ldots, b_{N}$ also exist.

Given $B_{0}, \ldots, B_{N}$-operators on a Hilbert space satisfying conditions (1)(5), we see (taking into account (3)) that on $\operatorname{ker} B_{0}$ all of them are equal to zero and the same holds for the adjoints (from (5)). The operators restricted to the orthogonal complement of $\operatorname{ker} B_{0}$ fulfill all the assumptions of Lemma 1. By virtue of that lemma we get a representation $U$ of $\mathbf{G}$ such that $\overline{d U\left(X_{m}\right)}=$ $\overline{B_{m} B_{0}^{-1}}$. It follows from the above considerations that $U$ extended to $C^{*}(\mathbf{G})$ must carry $b_{m}$ into $B_{m}$.

It remains to show that $b_{0}, \ldots, b_{N}$ generate $C^{*}(\mathbf{G})$. If that is not so, we get two different representations $\pi_{1}, \pi_{2}$ of $C^{*}(\mathbf{G})$ (acting on the same Hilbert space) which are identical on $b_{0}, \ldots, b_{N}$. From Lemma 1 we know that $\pi_{1}\left(b_{0}\right), \ldots, \pi_{1}\left(b_{N}\right)$ gives rise to a representation of the group. That is why $\pi_{1}$ and $\pi_{2}$ as the corresponding representations of the algebra must coincide. This completes the proof. Q.E.D.

Now we are able to complete the proof of the main Theorem.

Let $B^{m}:\left(B_{0}, \ldots, B_{N}\right) \mapsto B_{m}$ be the coordinate functions on $\mathbf{D}$. By virtue of [1, Theorem 3.4] $C_{0}(\mathbf{D})$ is generated by $B^{0}, \ldots, B^{N}$. It is an immediate consequence of Lemma 2 that the mapping $b_{m} \mapsto B^{m}$ can be extended to a *-homomorphism $\Gamma$ of $C^{*}(\mathbf{G})$ onto $C_{0}(\mathbf{D})$ (to see that one must apply part 1 of the lemma to the images of $B^{0}, \ldots, B^{N}$ under a faithful representation of $\left.C_{0}(\mathbf{D})\right)$.

Clearly $\Gamma$ has a trivial kernel. Indeed, if $\Gamma(c)=0$ then for any cyclic representation $\pi$ of $C^{*}(\mathbf{G})$ (acting necessarily on a separable Hilbert space $\mathscr{H}_{\pi}$ ) we have $\pi(c)=\Gamma(c)\left(\pi\left(b_{0}\right), \ldots, \pi\left(b_{N}\right)\right)=0$ (of course $\left(\pi\left(b_{0}\right), \ldots, \pi\left(b_{N}\right)\right) \in$ $\left.\mathbf{D}\left(\mathscr{H}_{\pi}\right)\right)$.

The proof is complete. Q.E.D.

\section{REFERENCES}

1. P. Kruszynski and S. L. Woronowicz, A noncommutative Gelfand-Naimark theorem, J. Operator Theory 8 (1982), 361-389.

2. E. Nelson, Analytic vectors, Ann. of Math. 70 (1959), 572-615. 
3. E. Nelson and W. F. Stinespring, Representation of elliptic operators in an enveloping algebra, Amer. J. Math. 81 (1959), 547-560.

4. G.K. Pedersen, $C^{*}$-algebras and their automorphism groups, Academic Press, London-New York-San Francisco, 1979.

5. G. Warner, Harmonic analysis on semi-simple Lie group, vol. I, Springer-Verlag, BerlinHeidelberg-New York, 1972.

Mathematics Institute, Polish Academy of Sciences, Sniadeckich 8, Warszawa, Poland 